\title{
Understanding Distributions of Environmental Parameters for Thermal Comfort Study in Singapore
}

\author{
Divya Periyakoil \\ divyap@berkeley.edu \\ University of California, Berkeley \\ Berkeley, California
}

\author{
Hari Prasanna Das \\ hpdas@berkeley.edu \\ University of California, Berkeley \\ Berkeley, California
}

\author{
Costas J. Spanos \\ spanos@berkeley.edu \\ University of California, Berkeley \\ Berkeley, California
}

\begin{abstract}
The National Science Experiment (NSE) is a nationwide project that began in 2015 in Singapore. As a part of this project, school students in Singapore carry the wearable device SENSg which measures environmental data such as temperature, humidity, light intensity, ambient noise, and air pressure. In this study, we analyzed NSE data collected during the year 2016 and examined how factors such as mode of transportation, location, and academic performance relate with various environmental parameters. We studied the relationships between environmental parameters such as temperature, humidity and noise with location, mode of transport, time, and student academic achievement. We studied three out of the five transportation modes, those that involved the user action in directly interacting with the corresponding settings (AC settings, humidity, etc.): not walking, walking, and traveling by car. We clustered them corresponding to a particular mode and by location in order to gauge mode specific and geographical patterns of environmental parameters in Singapore. For these first two tasks, we used the Expectation Maximization Algorithm in order to fit a n-component Gaussian Mixture Model to each of the three smaller datasets-where $\mathrm{n}$ was dependent on the mode of transportation or the geographical location. Finally, we compiled a list of the top 150 highest ranked secondary schools in Singapore in order to identify patterns of thermal comfort in schools with high rankings. We envision that the results of this study will provide insight into distribution of environmental conditions across Singapore.
\end{abstract}

\section{CCS CONCEPTS}

- Human-centered computing $\rightarrow$ Empirical studies in collaborative and social computing.

\section{KEYWORDS}

Temperature, Humidity, Noise, Environmental Parameters, Thermal Comfort, National Science Experiment, Singapore, Clustering

ACM Reference Format:

Divya Periyakoil, Hari Prasanna Das, and Costas J. Spanos. 2020. Understanding Distributions of Environmental Parameters for Thermal Comfort Study in Singapore. In The Eleventh ACM International Conference on Future

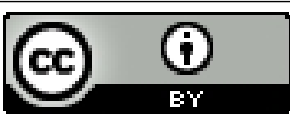

This work is licensed under a Creative Commons Attribution International 4.0 License.

e-Energy '20, fune 22-26, 2020, Virtual Event, Australia

(c) 2020 Copyright held by the owner/author(s).

ACM ISBN 978-1-4503-8009-6/20/06.

https://doi.org/10.1145/3396851.3402366
Energy Systems (e-Energy '20), June 22-26, 2020, Virtual Event, Australia. ACM, New York, NY, USA, 5 pages. https://doi.org/10.1145/3396851.3402366

\section{INTRODUCTION}

A country with a very warm, tropical climate, Singapore is dependent on consistent air conditioning usage in order to maintain the thermal comfort of its residents. About one third of the electricity produced in Singapore is used in buildings [3], and a large part of this electricity is used for space cooling $[4,6]$. However allocation of funds for public space cooling is inconsistent with the thermal comfort needs of the public. While most public buses and trains in Singapore provide air conditioning, this is not the case in most schools [9] [7]. The government has sought to cut funding totally for air conditioning in public schools, while issuing budget cuts for funding for air conditioning in independent schools [7]. We conducted this study to gain more insight into environmental patterns in Singapore, and how they may be affected by geography and mode of transportation, as well as how these patterns may be affecting academic performance.

\subsection{Data Collection}

The data used in this study were obtained from the National Science Experiment (NSE) and were collected through wearable devices worn by school students in Singapore. The National Science Experiment (NSE) is a nationwide project that began in 2015 in Singapore [14]. To collect data for this project, school students in Singapore carry the wearable device SENSg which collects environmental data such as temperature, humidity, light intensity, ambient noise, and air pressure [6] [15] [14] [12]. The data we used were exclusively collected in the year 2016. Prior research shows these environmental parameters play a crucial role in thermal comfort [10].

\subsection{Objective}

The objective of our work was to accurately model and infer environmental patterns-especially the thermal conditions-in Singapore. Specifically, we focused on how factors such as mode of transportation, location, and academic performance relate with various environmental parameters. We believe that the results of this project will provide insight into study of thermal comfort and related areas. Section 3 provides an in-depth explanation of the data processing and clustering methods that were used in this project. Section 4 presents the results and Section 5 discusses future works.

\section{RELATED WORK}

Previous work in the field of thermal comfort has noted the importance of appropriate air conditioning and ventilation in tropical climates and have sought to reduce thermal comfort (air-conditioning 
and ventilation) costs. Authors in [4] noted that heating, ventilation, and air-conditioning can consume more than $50 \%$ of the total energy consumption of a building. Additionally, a thermal comfort study conducted by [17] in Singapore from 2010-2011 analyzed thermal comfort perceptions and preferences of 2059 respondents from 13 outdoor spaces in Singapore. The study found that the most highly preferred temperature is $26.5\left({ }^{\circ} \mathrm{C}\right)$, with a tolerable range of 26.3-31.7 $\left({ }^{\circ} \mathrm{C}\right)$. These findings indicate the need for consistent $\mathrm{AC}$ usage in order to maintain thermal comfort. Studies also indicate that access to $\mathrm{AC}$ and the consequent thermal comfort can also have an effect on one's sleep environment, and, thus, sleep quality. For example, a study conducted by [16] concluded that a good sleeping environment is essential to maintain sound health and productivity.

Cardin [2] and Happle [6] have used the NSE data for studying environmental parameters. Cardin used the environmental data collected by the NSE project in order to learn more about how people travel, what modes of transportation they prefer, and which activities they travel to and from [2]. Happle used the NSE data to model AC usage patterns in Singapore [6]. In previous work [6], it was assumed that humidity and temperature measurements are independent, although humidity readings were dependent on the temperature, which lead to uncertainty in the distributions that temperature and humidity readings were assumed to have been drawn from. We formulated each of the components of the Gaussian Mixture Model (GMM) for clustering with their own general covariance matrix, lending more noise, thus improving accuracy of our results. Additionally, we performed analysis on more features: temperature, humidity, and noise, lending more clear insight into thermal comfort as governed by these parameters [11].

\section{METHODS}

\subsection{Data Pre-processing}

We applied a number of filtering criteria to the dataset [6]: (1) minimum temperature less than $40^{\circ} \mathrm{c}$ in every hour; (2) minimum increase of 300 count of steps during school hours (considered as $8 \mathrm{am}-5 \mathrm{pm})$; (3) at least one data point in every hour; and a (4) minimum difference of $15 \%$ between the highest and lowest observed humidity reading in the 24 hours. We selected 3 features from the data: temperature $\left({ }^{\circ} \mathrm{C}\right)$, humidity (\% rh), and noise (decibels). We then normalized the data using min-max normalization.

\subsection{Clustering based on Transportation Mode via Gaussian Mixture Model}

We divided the pre-processed dataset into 3 parts corresponding to 3 chosen modes of transportation: not walking, walking, and car. We applied the Expectation Maximization (EM) Algorithm via an ncomponent Gaussian Mixture Model to each of the smaller datasets. The " $n$ "was picked depending on the typical physical states of environmental patterns for that mode of transportation. We clustered the datasets corresponding to "not walking "and "walking"with 2-component GMMs. We clustered the dataset corresponding to "car"with a 3-component GMM.

\subsection{Clustering Location Dependent Datasets via Gaussian Mixture Model}

We performed analysis on the relationship between geographical location and thermal comfort. We first identified the northern (Sembawang), southern (Sentosa Cove), eastern (Changi Village), and western (Jurong West) most residential points of Singapore. We then marked the center of Singapore to be the center of the four extreme points that we identified. We picked four points to be our geographical cluster centers. These points were the points situated directly in between the the center and the northern, southern, eastern, and western most points. We then assigned each point in the dataset to one of the four groups, depending on which cluster was the shortest euclidean distance away from the point and studied the distributions of temperature over time for each group.

\subsection{Determining Patterns of Environmental Parameters with Academic Performance}

We analyzed the relationship between thermal comfort and high school academic performance. We took a list of the top 150 ranked secondary schools in Singapore [1] and located each school in terms of latitude and longitude coordinates. For each school we filtered the dataset to retrieve all points within a radius of 0.005 decimal units from the the school in terms of latitude and longitude. We took the average temperature of all of these points, and assigned this to be the average temperature of the school. We used these average temperature values to study how academic performance relates to the environmental parameters.

\section{RESULTS}

\subsection{Data Filtering}

We started with a dataset of nearly 100 million data points, and we sampled 10 million of the data points. After we applied the filtering criteria from Section 3.1 to the data 1921744 data points remained, spanning fifty-eight days. We separated the data points by mode of transportation, which resulted in 5 data sets: 1361730 "not walking”, 237732 "walking”, 62105 "train”, 17644 "bus”, and 242533 "car”. Among "bus", "train"and "car", we consider only those modes of transportation which allow the individual direct control and access to changing thermal comfort settings of their environment, i.e. car.

\subsection{Transportation Mode based Clustering}

We applied the Expectation Maximization Algorithm via an ncomponent-where $\mathrm{n}$ was dependent on the mode of transportationGaussian Mixture Model to data from each of the three modes of transportation. For the modes "not walking" and "walking", we used a 2-component and for the mode "car"we used a 3-component GMM.

For the mode "not walking" and "walking"-as shown in Fig. 1 and Fig. 2 respectively, the first center had higher temperature, humidity, and noise than the second. For "not walking", the blue cluster is centered at (T:31.7, H:63.4, N:49.9), while the red cluster is centered at (T:33.3, H:68.6, N:63.4). For walking, the red cluster is centered at (T:31.1, H:64.4, N:63.5), while the blue cluster is centered at (T:33.3, H:66.7, N:68.3). For these two modes of transportation, we assigned the first cluster to the state of being outdoors, and the second cluster to the state of being indoors. 


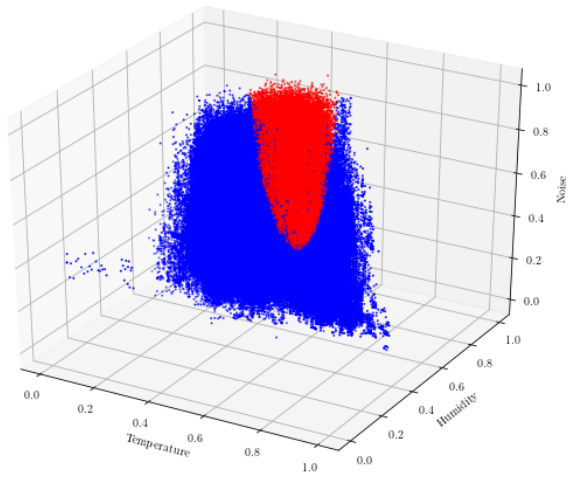

Figure 1: Clustering results for mode "not walking"

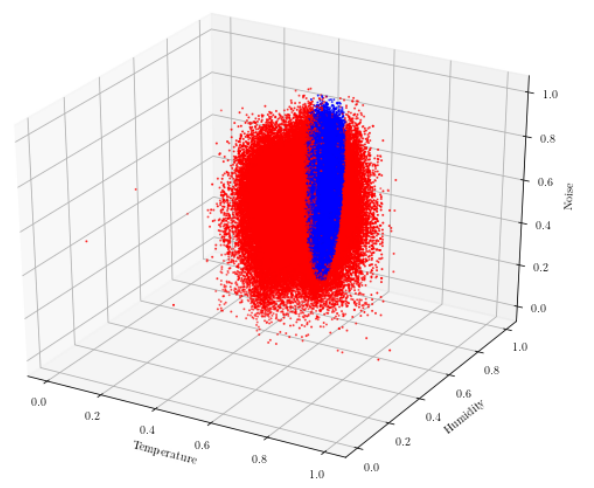

Figure 2: Clustering results for mode "walking"

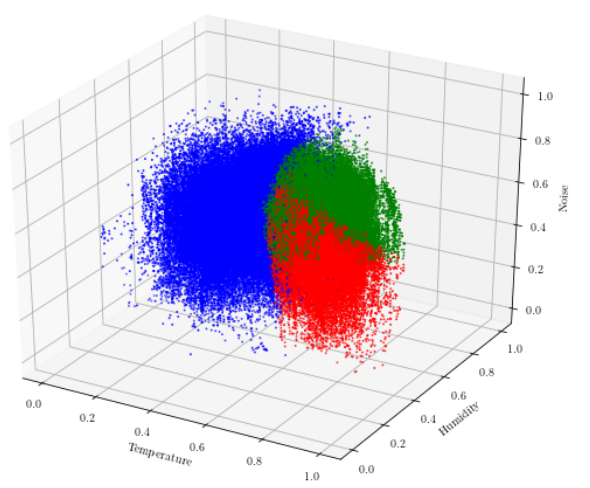

Figure 3: Clustering results for mode "car"

For the mode "car"-as shown in Fig. 3, cluster 1 (green) center has high temperature, humidity, and noise (T:35.8, H:63.7, N:67.9). We assigned this cluster to the state of having the window open. Cluster 2 (red) has a high temperature, high humidity, but low noise (T:35.2, H:65.6, N:57.2). We assigned this cluster to the state of having the window closed and the AC off. Cluster 3 (blue) has lower temperature, low humidity, and higher noise than cluster 2, but lower than cluster 1 (T:29.9, H:63.4, N:61.5). We assigned this cluster to the state of having the $\mathrm{AC}$ on but the window closed.

\subsection{Transportation Mode based Distribution}

We also plotted the distribution of temperature across time for each of the modes of transport. For the mode "not walking"-as shown in Fig 4, we noted that variation in temperature was quite standard during school hours (8am-4pm) and that there was higher variation in temperature during non-school hours (12am-7am and $5 \mathrm{pm}-11 \mathrm{pm}$ ). This was presumably because schools which have AC have a standard AC setting, while students experience various temperature settings when they are at home, depending upon their different living circumstances and household practices. The median temperature throughout the day remained relatively constant, probably because when a student is seated for a prolonged period of time with no movement, the data acquisition is hampered as per the design of the original experiment.

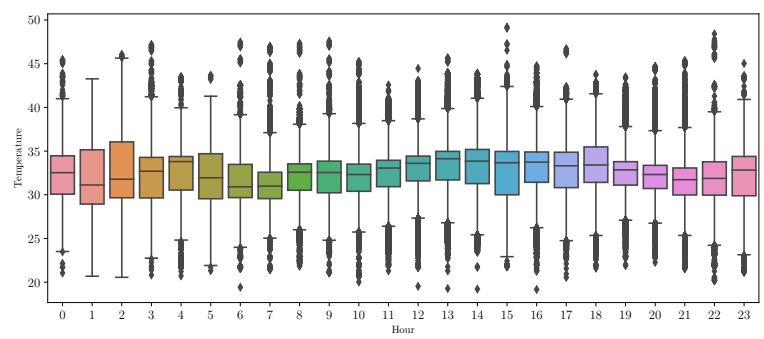

Figure 4: Distribution of temperature over time for mode "not walking"

For the mode "walking"-as shown in Fig. 5, the median temperature remained consistent throughout the day (between 30 and 35 ${ }^{o} \mathrm{C}$ ), likely because people are not stationary (sleeping or in class), meaning that they have no reason to change their temperature settings. There was a greater variation in temperature during the early hours of the morning, but this could be attributed to keeping the $\mathrm{AC}$ on throughout the night or just at the beginning of the night. It could also be attributed to naturally lower temperatures at night.

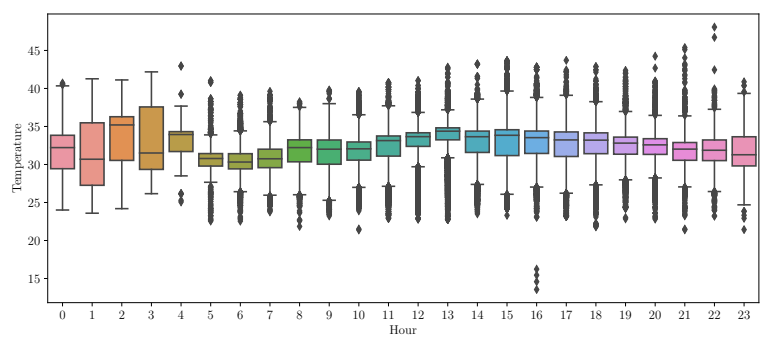

Figure 5: Distribution of temperature over hours of the day for mode: "walking" 
For the mode "car", as shown in Figure 6, we observed that temperature was extremely variable throughout the day. This could be because individuals have varying preferences for temperature as well as direct control over the AC settings of their car. The period of interest here includes the time excluding $8 \mathrm{AM}$ to $5 \mathrm{PM}$ when the students are in school. The range of temperature in this dataset was $[21.55,43.86]^{\circ} \mathrm{C}$. The observation of a wide range of temperature measurements is consistent with the existence of three clusters within the dataset.

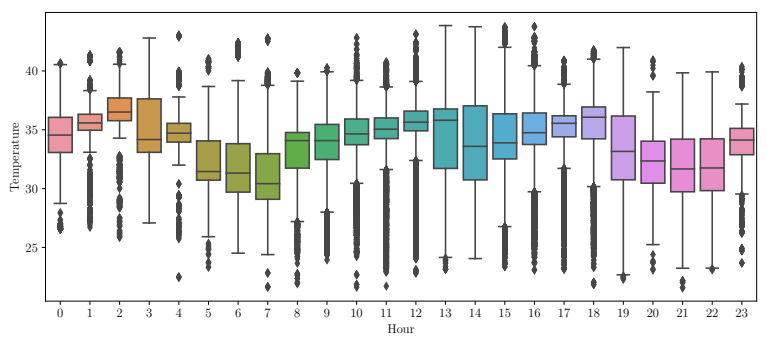

Figure 6: Distribution of temperature over hours in the day for mode: "car"

\subsection{Location Dependent Analysis}

We applied a 2-component GMM to each of the four groups corresponding to north, south, east, and west. We found that the cluster centers for south and west were characterized by low temperatures while the cluster centers for east and north were characterized by high temperatures. Humidity and noise appeared uniform across location. We wished to examine if there was any specific reason why certain locations in Singapore were characterized by lower average temperatures as opposed to others, and found that the south and east are home to some of the wealthiest residential areas of Singapore such as Sentosa Cove, Bukit Timah, and Tanglin [13].

\subsection{Relationship Between Thermal Comfort and Academic Performance}

In order to determine the relationship between thermal comfort and academic performance, we collated a list of the the top 150 secondary schools in Singapore. We made two findings about the top ranked high schools in Singapore. First, we estimated the average temperatures of these schools and found that every school's temperature was consistently in the twenties and low thirties. The range of temperatures was [27.8, 35.3]. This range of temperatures is quite high, and is not indicative of consistent AC usage. These findings corroborated reports made in section 1 and inspired further questions about how air conditioning in schools may be affecting academic performance, reminiscent of the research by Yang and Olofsson on how access to air conditioning during sleep can affect productivity and performance [16].

Next, we found that although average temperatures vary with geographic location (as shown in the previous section), thermal comfort in top schools remained relatively unvaried, regardless of location. To demonstrate this, we plotted the latitude and longitude

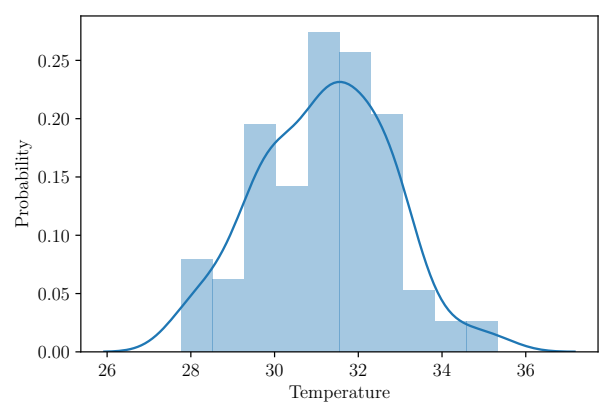

Figure 7: Distribution of Temp. in Top 150 High Schools

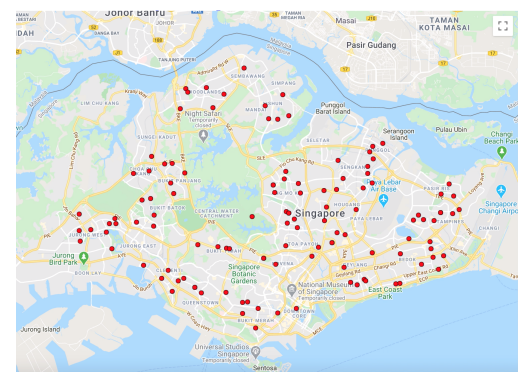

Figure 8: Locations of Top 150 Schools in Singapore

values of the full dataset and those of the schools, and found that these top schools were relatively uniformly distributed around Singapore, as shown in Figure 8.

\section{CONCLUSION AND FUTURE WORKS}

In this work, we analyzed the environmental parameters pattern across Singapore based on the dataset from NSE experiment.We separated our dataset by mode of transportation and clustered each of the datasets corresponding to a particular mode individually. This flexibility provided by certain modes of transportation-such as car-had high variability and could have an effect on the environmental settings. We made our results more accurate, by formulating each of the components of the GMM corresponding to each mode of transportation with their own general covariance matrix, lending more noise. Our work on exploring relation between location and environmental parameters showed patterns across geography of Singapore. Finally, our analysis of academic performance as it relates to thermal comfort showed that although average thermal comfort settings vary across geographical locations in Singapore, top schools maintain roughly uniform thermal comfort levels regardless of location. Our research can prove beneficial in performing an accurate thermal comfort analysis for tropical environments such as that of Singapore's.

Taking this work forward, we will explore how transfer learning techniques for built environment [18-20] can be utilized to generate synthetic distributions of environmental parameters for a range of variations of tropical environments. We will also incorporate the information into design of future social game experiments to save energy, as performed in our prior works $[5,8]$. 


\section{ACKNOWLEDGMENTS}

This research is funded by the Republic of Singapore's National Research Foundation through a grant to the Berkeley Education Alliance for Research in Singapore (BEARS) for the SingaporeBerkeley Building Efficiency and Sustainability in the Tropics (SinBerBEST) Program. BEARS has been established by the University of California, Berkeley as a center for intellectual excellence in research and education in Singapore. We would also like to acknowledge the organizers of National Science Experiment, Singapore for providing the data used in this research.

\section{REFERENCES}

[1] Bryan. 2019. Top Secondary Schools (2019 cut-off). https://www.salary.sg/2019/ top-secondary-schools-2019-cut-off/

[2] Michel-Alexandre Cardin, Saik Hay Fong, Daniel Krob, Lui Pao Chuen, and Yang How Tan. 2016. Complex Systems Design \& Management Asia: Smart NationsSustaining and Designing: Proceedings of the Second Asia-Pacific Conference on Complex Systems Design \& Management, CSD\&M Asia 2016. Vol. 426. Springer.

[3] K.J. Chua and S.K. Chou. 2010. Energy performance of residential buildings in Singapore. Energy 35, 2 (2010), 667 - 678. https://doi.org/10.1016/j.energy.2009. 10.039 ECOS 2008.

[4] K.J. Chua, S.K. Chou, W.M. Yang, and J. Yan. 2013. Achieving better energyefficient air conditioning - A review of technologies and strategies. Applied Energy 104, C (2013), 87-104. https://doi.org/10.1016/j.apenergy.2012.1

[5] Hari Prasanna Das, Ioannis C Konstantakopoulos, Aummul Baneen Manasawala, Tanya Veeravalli, Huihan Liu, and Costas J Spanos. 2019. A Novel Graphical Lasso based approach towards Segmentation Analysis in Energy Game-Theoretic Frameworks. arXiv preprint arXiv:1910.02217 (2019).

[6] Gabriel Happle, Erik Wilhelm, Jimeno A. Fonseca, and Arno Schlueter. 2017. Determining air-conditioning usage patterns in Singapore from distributed, portable sensors. Energy Procedia 122 (2017), 313 - 318. https://doi.org/10.1016/j.egypro. 2017.07.328

[7] Linette Heng and Jasmine Lim. 2016. Air-con in school not so cool any more. https://www.tnp.sg/news/others/air-con-school-not-so-cool-any-more

[8] Ioannis C Konstantakopoulos, Hari Prasanna Das, Andrew R Barkan, Shiying He, Tanya Veeravalli, Huihan Liu, Aummul Baneen Manasawala, Yu-Wen Lin, and Costas J Spanos. 2019. Design, Benchmarking and Explainability Analysis of a Game-Theoretic Framework towards Energy Efficiency in Smart Infrastructure.
arXiv preprint arXiv:1910.07899 (2019)

[9] Everlyn Lee. [n.d.]. Public Transportation in Singapore. https://worksingapore. com/articles/live_4.php

[10] S. Liu, M. Jin, H. Das, C. Spanos, and S. Schiavon. 2018. Personal thermal comfort models based on physiological parameters measured by wearable sensors. Proceedings of the Windsor Conference (2018), 431-441.

[11] Shichao Liu, Stefano Schiavon, Hari Prasanna Das, Ming Jin, and Costas J. Spanos. 2019. Personal thermal comfort models with wearable sensors. Building and Environment 162 (2019), 106281. https://doi.org/10.1016/j.buildenv.2019.106281

[12] Harry J. Sauer, Ronald H. Howell, and William J. Coad. 2001. Principles of heating, ventilating, and air conditioning: a textbook with design data based on the 2001 ASHRAE handbook-Fundamentals. American Society of Heating, Refrigerating and Air-Conditioning Engineers.

[13] Katie Warren. 2019. What it's like living as a billionaire in Singapore, the most expensive city in the world, where wealthy residents are worth a combined 1 trillion and limited land makes owning a house the ultimate 'status symbol'. https://www.businessinsider.com/how-singapore-billionaires-live-lifestylemansions-social-clubs-private-schools-2019-2\#one-of-the-most-prestigiousis-the-anglo- chinese-school-founded-in-1886-where-tuition-can-be-as-lowas-3250-a-year-for-residents-and-up-to-22000-for-foreign-students- 44

[14] E. Wilhelm, S. Siby, Y. Zhou, X. J. S. Ashok, M. Jayasuriya, S. Foong, J. Kee, K. L. Wood, and N. O. Tippenhauer. 2016. Wearable Environmental Sensors and Infrastructure for Mobile Large-Scale Urban Deployment. IEEE Sensors fournal $16,22(2016), 8111-8123$.

[15] Erik Wilhelm, Nils Ole Tippenhauer, Yuren Zhou, and Nan Zhang. 2016. SENSg: Large-Scale Deployment of Wearable Sensors for Trip and Transport Mode Logging.

[16] Bin Yang and Thomas Olofsson. 2015. A questionnaire survey on sleep environment conditioned by different cooling modes in multistorey residential buildings of Singapore. Indoor and Built Environment (08 2015). https: //doi.org/10.1177/1420326X15604206

[17] Wei Yang, Nyuk Hien Wong, and Steve Kardinal Jusuf. 2013. Thermal comfort in outdoor urban spaces in Singapore. Building and Environment 59 (2013), 426 435. https://doi.org/10.1016/j.buildenv.2012.09.008

[18] Han Zou, Hari Prasanna Das, Jianfei Yang, Yuxun Zhou, and Costas Spanos. 2019. Machine Learning empowered Occupancy Sensing for Smart Buildings. (2019).

[19] Han Zou, Jianfei Yang, Hari Prasanna Das, Huihan Liu, Yuxun Zhou, and Costas Spanos. 2019. WiFi and Vision Multimodal Learning for Accurate and Robust Device-Free Human Activity Recognition. In Proceedings of the IEEE Conference on Computer Vision and Pattern Recognition Workshops. 0-0.

[20] H. Zou, Y. Zhou, J. Yang, H. Liu, H.P. Das, and C.J. Spanos. 2019. Consensus Adversarial Domain Adaptation. In AAAI Conference on Artificial Intelligence. 